\title{
Hémorragie cérébrale et spot sign
}

\section{Spot Sign in Intracerebral Hemorrhage}

\section{R. Girerd · E. Beti}

Reçu le 20 avril 2017; accepté le 22 mai 2017

(C) SFMU et Lavoisier SAS 2017
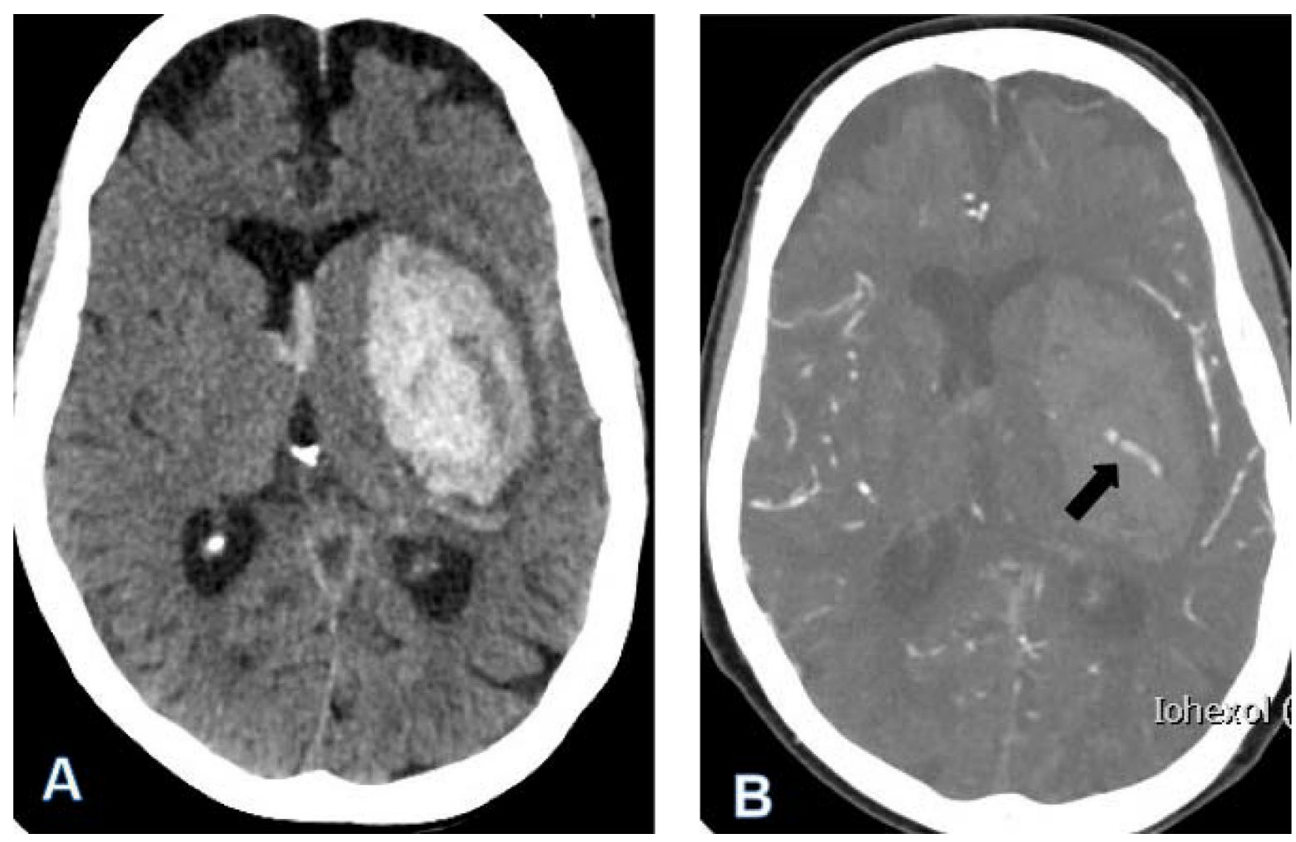

Fig. 1 A : scanner cérébral sans injection : hématome cérébral intraparenchymateux profond gauche avec hémorragie méningée sylvienne gauche et contamination ventriculaire ; B : angioscanner cérébral montrant un spot sign (flèche noire) : extravasation de produit de contraste dans l'hématome intraparenchymateux

Une patiente de 60 ans, hypertendue, est admise aux urgences pour un déficit hémicorporel droit apparu brutalement $1 \mathrm{~h} 30$ auparavant. À son arrivée, la tension artérielle est $151 / 78 \mathrm{mmHg}$, le pouls à 64 pulsations/min, la température à $36^{\circ} \mathrm{C}$, la glycémie capillaire $1,52 \mathrm{~g} / \mathrm{L}$, la saturation en oxygène à $97 \%$ en air ambiant. L'électrocardiogramme s'inscrit en rythme sinusal. Le score de Glasgow est à 11 du côté gauche. Le score de NIHSS est coté à 20. Une tomodensitométrie cérébrale sans injection est réalisée, montrant un hématome cérébral profond gauche évaluée à $45 \mathrm{~mm}^{3}$ associé à une hémorragie méningée (Fig. 1A). Un angioscanner

R. Girerd $(\bowtie) \cdot$ E. Beti

Service des urgences, centre hospitalier Gabriel Martin,

18 rue Labourdonnais, F-97460 Saint Paul, La Réunion, France e-mail : remigirerd@gmail.com cérébral (Fig. 1B) montre une fuite de produit de contraste dans l'hématome, traduisant donc un saignement actif, appelé spot sign. Il n'y a pas d'anévrysme ou de malformation artérioveineuse. Une dégradation neurologique conduit à une prise en charge neurochirurgicale (évacuation de l'hématome). L'évolution est très lentement favorable, le score de Rankin est à cinq. Le spot sign est un signe de gravité prédictif d'une expansion hémorragique, de mauvais pronostic neurologique, et de mortalité [1].

\section{Référence}

1. Golstein JN, Fazen LE, Snider R, Schwab K, et al (2007) Contrast extravasation on $\mathrm{CT}$ angiography predicts hematoma expansion in intracerebral hemorrhage. Neurology 12:889-94 